\title{
A Network-Based Approach to Explore the Mechanism and Bioactive Compounds of Erzhi Pill against Metabolic Dysfunction- Associated Fatty Liver Disease
}

\author{
Shaojie Huang $\mathbb{D}$, Fei Mu $\mathbb{D}$, Fei Li, Wenjun Wang, Haixia Chen, Lu Lei, Yang Ma, Yi Ding $\mathbb{D}$, \\ and Jingwen Wang $\mathbb{B}$
}

Department of Pharmacy, Xijing Hospital, Fourth Military Medical University, Xi'an, 710032, China

Correspondence should be addressed to Yi Ding; dingyi.007@163.com and Jingwen Wang; wangjingwen8021@163.com

Received 3 June 2020; Accepted 18 June 2020; Published 17 July 2020

Academic Editor: Hiroshi Okamoto

Copyright (C) 2020 Shaojie Huang et al. This is an open access article distributed under the Creative Commons Attribution License, which permits unrestricted use, distribution, and reproduction in any medium, provided the original work is properly cited.

Erzhi pill (EZP), a classical traditional Chinese medicine prescription, exerts a potent hepatoprotective effect against metabolic dysfunction-associated fatty liver disease (MAFLD), previously known as nonalcoholic fatty liver disease (NAFLD). However, the mechanism and bioactive compounds underlying the hepatoprotective effect of EZP have not been fully elucidated. In this study, a systematic analytical platform was built to explore the mechanism and bioactive compounds of EZP against MAFLD. This was carried out through target prediction, protein-protein interaction (PPI) network construction, gene ontology, KEGG pathway enrichment, and molecular docking. According to the topological parameters of the PPI network, compound-targetpathway network, 9 targets, and 11 bioactive compounds were identified as core targets and bioactive compounds for molecular docking. The results showed that EZP exerts anti-MAFLD effects through a multicomponent, multitarget, multipathway manner, and luteolin and linarin may be the bioactive compounds of EZP. This study provides further research insights and helps explore the hepatoprotective mechanism of EZP.

\section{Introduction}

Metabolic dysfunction-associated fatty liver disease (MAFLD), previously known as nonalcoholic fatty liver disease (NAFLD) [1,2], is defined as the presence of liver cells with steatosis exceeding $5 \%$ and the lack of secondary causes of liver fat accumulation, such as drinking of alcohol [3]. With dramatic lifestyle modifications, the MAFLD has developed into a global health concern over the past decades [4]. Moreover, studies have increasingly shown the multisystem disease nature of MAFLD which affects several organs and increases the risk of type 2 diabetes and cardiovascular, cardiac, and chronic kidney diseases $[5,6]$. Significant weight loss and change of dietary habits will have a salutary effect on MAFLD; however, new treatment strategies are urgently needed [7]. The reason is that with the changing dietary habits and lifestyle, MAFLD is one of the most important causes of liver disease worldwide. More importantly, MAFLD may eventually become the primary cause of end-stage liver disease [4]. Therefore, there is an urgent need for safe and effective drugs against MAFLD.

Previous studies have shown that some traditional Chinese medicine (TCM) formulaes, such as Dachaihu decoction, have good efficacy against MAFLD [8]. Erzhi pill (EZP) is a TCM used for liver disease in the past centuries. EZP consists of Ligustri Lucidi Fructus (LLF) and Ecliptae Herba (EH) at a ratio of $1: 1$ and functions as a liver and kidney tonic in traditional Chinese medicine theory. A previous study showed the hepatoprotective effect of EZP by the antioxidative defense system enhancement and the inflammatory response through the TSC/mTOR signaling pathway [9]. EZP has also been used to treat diabetes and metabolic syndrome. However, studies on the mechanism of EZP against MAFLD are still lacking.

The network pharmacology presented in 2008 [10] has holistic and systematic research methods and characteristics 
of focusing on the interaction between drugs and the body system. This is consistent with the characteristics of multiple targets and multiple pathways in TCM [11], becoming an efficient tool to systematically analyse the multiple targets and multiple pathway mechanisms of TCM. Several studies that employed network pharmacology to investigate the mechanism of TCM have been successful $[12,13]$. In addition, the interaction of compounds, targets, and pathways can be established with network pharmacology, which helps identify potential bioactive compounds and pathways of TCM.

In this study, a systematic analytical platform for predicting potential bioactive compounds, targets, and molecular mechanisms of EZP against MAFLD was built. Detailed methods included potential bioactive compound collection, EZP- and MAFLD-related target prediction, protein-protein interaction (PPI) network construction, gene ontology and pathway enrichment, and molecular docking. This study provides a further research direction for the exploration of the hepatoprotective mechanism of EZP.

\section{Materials and Methods}

2.1. Extraction of Bioactive Components of EZP. The date for EZP compounds were mainly obtained from the Traditional Chinese Medicine Systems Pharmacology Database (TCMSP, http://lsp.nwu.edu.cn/tcmsp.php, Version 2.3), a pharmacology platform that provides information on drugs, targets, and diseases, by retrieving Fructus Ligustri Lucidi and Ecliptae Herba. Twelve absorption, distribution, metabolism, and excretion- (ADME-) related parameters of herbal ingredients were also extraction from the TCMSP [14]. Considering that oral administration of EZP, OB [15], and DL [16] was used for identifying bioactive compounds of EZP, the components with $\mathrm{OB} \geq 30 \%$ and $\mathrm{DL} \geq 0.18$ were identified as potential bioactive compounds.

2.2. Collection of Potential Targets of the Bioactive Compounds of EZP. Bioactive compound-related targets were obtained from PharmMapper (http://lilab-ecust.cn/ pharmmapper/, Version 2017), TargetNet (http://targetnet .scbdd.com/home/index/), and Swiss Target Prediction (http://www.swisstargetprediction.ch/, 2019 version) [17-22]. These platforms achieved compound-related targets by adopting various prediction algorithms. First, information on the bioactive compounds was collected from PubChem (https:// pubchem.ncbi.nlm.nih.gov/) and TCMSP, including molecular structures in mol2 format and canonical smiles. To predict targets of compounds, molecular structures in mol2 were uploaded to PharmMapper with limitation to "Homo sapiens" and a normal fit score $\geq 0.6$. In addition, canonical smiles were uploaded to Swiss Target Prediction and TargetNet with limitation to "Homo sapiens" (in Swiss Target Prediction), AUC $\geq 0.7$ (in TargetNet), and probability $\geq 0.6$. Finally, all the targets were transferred to UniProtKB (https://www.uniprot.org/) to avoid mix-up across the databases and platforms.

2.3. Construction of the MAFLD Target Database. Considering the different advantages and characteristics of each database, four databases were used to collect the MAFLDrelated targets. By retrieving "nonalcoholic fatty liver disease" in GeneCards (https://www.genecards.org/), DrugBank (https://www.drugbank.ca/, version 5.1.5), Online Mendelian Inheritance in Man (OMIM, http://omim.org/, updated on Jan. 15, 2019), and National Centre for Biotechnology Information Gene (NCBI Gene, https:/www.ncbi.nlm.nih.gov/ gene/) MAFLD-related targets were retrieved. All the four databases are freely accessible platforms that contain comprehensive molecular information about drugs, targets, targets related to disease, gene function, etc. and can be used to collect targets related to the disease [23-26]. To maintain the reliability of the target collection, only the targets approved by the FDA in DrugBank, norm fit scores higher than 20 in GeneCards or the species limited to "Homo sapiens" in the NCBI Gene were identified as MAFLD-related targets. Finally, the target names were standardized to the UniProtKB form and duplicates were removed.

2.4. Construction of Protein-Protein Interaction (PPI) Network. A PPI network was built and analyzed by Search Tool for the Retrieval of Interacting Genes (STRING, https://string-db.org/), which can be employed for the system-wide understanding of cellular function between the expressed proteins [27]. After removing the overlap section and standardizing target names, the intersection of bioactive compound-related targets and MAFLD-related targets were uploaded to STRING with limitations to "Homo sapiens" and a confidence score $>0.9$. The PPI network was constructed and visualized using Cytoscape 3.7.1, a software that is used for analyzing and visualizing biomolecular interaction networks [28].

2.5. Enrichment Analysis and Network Construction. Database for Annotation, Visualization, and Integrated Discovery (DAVID, https://david.nicifcrf.gov/, version 6.8) was used for enrichment analysis with the screening criteria of $P \leq$ 0.05 using Bonferroni correction [29]. Furthermore, KEGG Mapper (https://www.genome.jp/kegg/mapper.html) was employed for the analyses of upstream and downstream genes of the key signaling pathway [30, 31]. Thereafter, pathways with the top 20 protein numbers were used for the establishment of the compound-target-pathway network by Cytoscape.

2.6. Molecular Docking. Molecular docking was performed with AutoDock Tools [32] (version 1.5.6 http://mgltools .scripps.edu/). The 3D molecular structures of the bioactive compounds were collected from TCMSP in mol2 format and transformed into PDPQT format with AutoDock Tools. Protein Data Bank (PDB, http://www.rcsb.org/) was utilised for the collection of crystal structures of the core targets. AutoDock Tools were further used for removal of water and addition of hydrogen atoms to the crystal structures of core targets and saved as PDPQT format. Molecular docking between the bioactive compounds and core targets was performed with AutoDock. Finally, the binding pattern with the lowest binding energy was selected for further analysis. 
TABLE 1: A list of bioactive compounds of EZP for network analysis.

\begin{tabular}{|c|c|c|c|c|c|c|}
\hline Mol ID & Molecule name & MW & OB (\%) & $\mathrm{DL}$ & Pubchem ID & Herb \\
\hline MOL000006 & Luteolin & 286.25 & 36.16 & 0.25 & 5280445 & LLF/EH \\
\hline MOL000098 & Quercetin & 302.25 & 46.43 & 0.28 & 5280343 & LLF/EH \\
\hline MOL000263 & Oleanolic acid & 456.78 & 29.02 & 0.76 & 10494 & LLF \\
\hline MOL000358 & Beta-sitosterol & 414.79 & 36.91 & 0.75 & 222284 & LLF \\
\hline MOL000422 & Kaempferol & 286.25 & 41.88 & 0.24 & 5280863 & LLF \\
\hline MOL002929 & Salidroside & 300.34 & 7.01 & 0.20 & 159278 & LLF \\
\hline MOL004576 & Taxifolin & 304.27 & 57.84 & 0.27 & 439533 & LLF \\
\hline MOL005146 & Lucidumoside D & 568.63 & 48.87 & 0.71 & 10531060 & LLF \\
\hline MOL005147 & Lucidumoside D_qt & 406.47 & 54.41 & 0.47 & - & LLF \\
\hline MOL005169 & (20S)-24-ene-3 $\beta, 20$-diol-3-acetate & 486.86 & 40.23 & 0.82 & 185500 & LLF \\
\hline MOL005188 & Specnuezhenide & 686.73 & 19.30 & 0.50 & 91895359 & LLF \\
\hline MOL005190 & Eriodictyol & 288.27 & 71.79 & 0.24 & 440735 & LLF \\
\hline MOL005195 & Syringaresinol diglucoside_qt & 450.48 & 83.12 & 0.8 & 21603207 & LLF \\
\hline MOL005209 & Lucidusculine & 401.6 & 30.11 & 0.75 & 101286217 & LLF \\
\hline MOL005211 & Olitoriside & 696.87 & 65.45 & 0.23 & 94348 & LLF \\
\hline MOL005212 & Olitoriside_qt & 404.55 & 103.23 & 0.78 & - & LLF \\
\hline MOL001689 & Acacetin & 284.28 & 34.97 & 0.24 & 5280442 & $\mathrm{EH}$ \\
\hline MOL001790 & Linarin & 592.6 & 39.84 & 0.71 & 5317025 & $\mathrm{EH}$ \\
\hline MOL002975 & Butin & 272.27 & 69.94 & 0.21 & 28125525 & $\mathrm{EH}$ \\
\hline MOL003389 & $3^{\prime}$-O-Methylorobol & 300.28 & 57.41 & 0.27 & 5489605 & $\mathrm{EH}$ \\
\hline MOL003398 & Pratensein & 299.27 & 39.06 & 0.28 & 5319744 & $\mathrm{EH}$ \\
\hline MOL003402 & Demethylwedelolactone & 302.25 & 72.13 & 0.43 & 5281803 & $\mathrm{EH}$ \\
\hline MOL003404 & Wedelolactone & 314.26 & 49.6 & 0.48 & 5281813 & $\mathrm{EH}$ \\
\hline
\end{tabular}

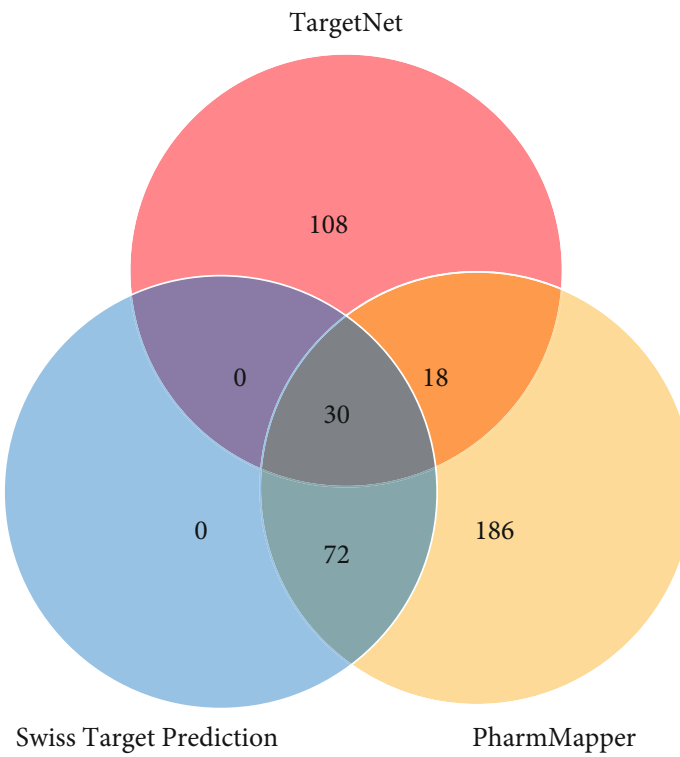

(a)

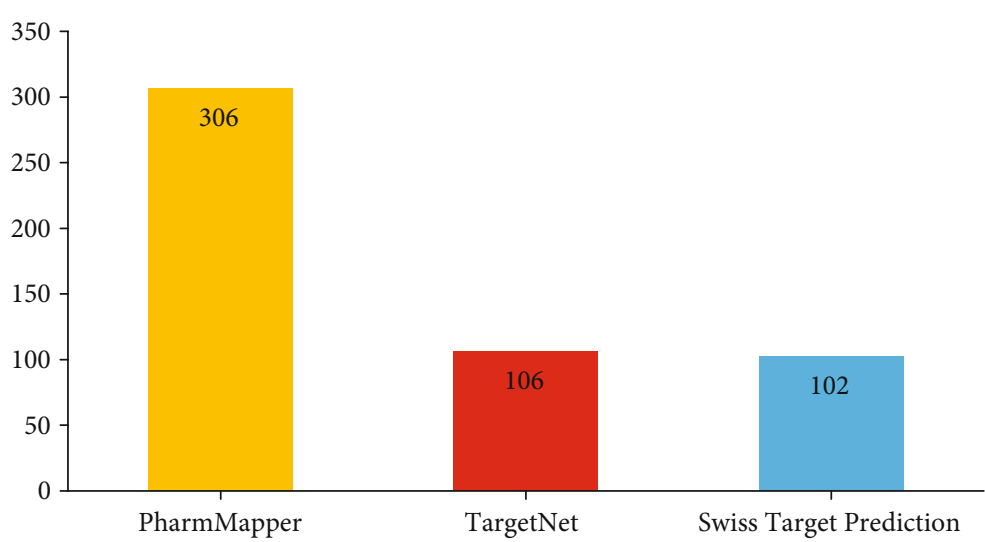

(b)

FIGURE 1: Analysis of predicted genes of potential bioactive compounds of EZP from 3 databases. (a) Venn graph showing the number of the overlapping genes from PharmMapper (yellow), TargetNet (red), and Swiss Target Prediction (blue). (b) The number of predicted genes from each database. 


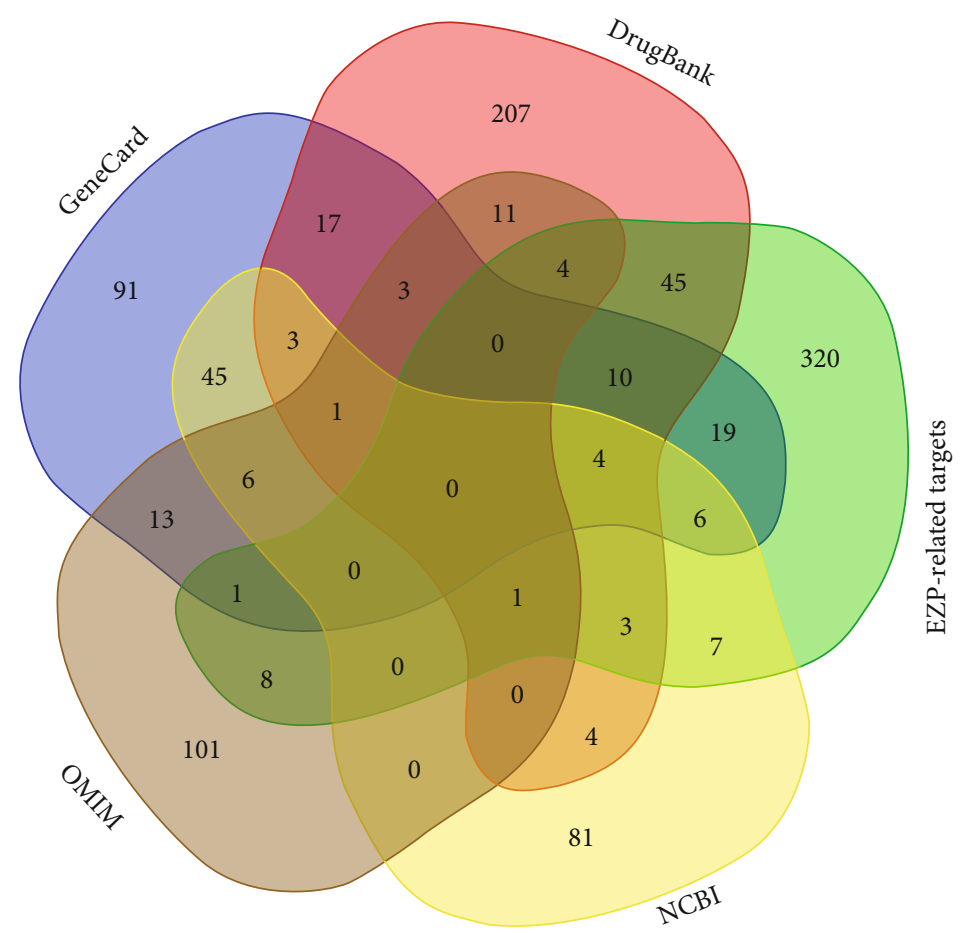

(a)

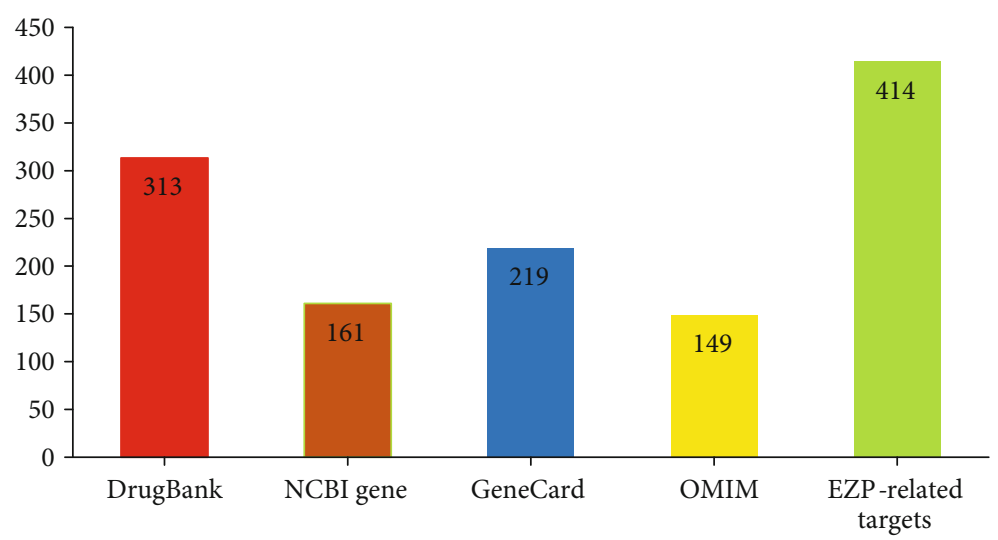

(b)

FIGURE 2: Analysis of predicted MAFLD genes. (a) Venn graph showing the numbers of overlapping genes from DrugBank (red), NCBI Gene (brown), GeneCard (blue), OMIM (yellow), and EZP-related targets (green). (b) The number of predicted genes from each database.

The interactions between the bioactive compounds and the core targets were visualized as 3D diagrams using PyMol 1.8.

\section{Result}

3.1. Bioactive Compounds in EZP. There were 166 compounds of EZP retrieved from TCMSP, including 47 in EH, and 119 in LLF, and 5 overlapping compounds were removed, resulting in 161 identified compounds. Finally, 20 bioactive compounds were identified after ADME screening with $\mathrm{OB} \geq 30 \%$ and $\mathrm{DL} \geq 0.18,13$ in LLF and 9 in $\mathrm{EH}$ ( 2 were duplicated and therefore removed). This is illustrated in Table 1. Some compounds that were removed after ADME screening have been identified as the main compounds of EZP in previous studies $[33,34]$. Therefore, oleanolic acid, salidroside, and specnuezhenide were identified as bioactive compounds.

3.2. Potential Target Prediction for Bioactive Compounds of EZP. To identify potential targets of the 23 bioactive compounds, Swiss Target Prediction, PharmMapper, and TargetNet were used to predict the bioactive compounds' targets. There were 306 targets from PharmMapper (norm fit >0.6), 156 targets from TargetNet (probability $>0.8$ ), and 102 targets from Swiss Target Prediction (probability $>0.8$ ) as shown in Figure 1(a). Finally, 30 targets were shared by all three databases, 72 targets were shared with Swiss Target Prediction and PharmMapper, and 18 targets were shared with PharmMapper and TargetNet (Figure 1(b)). After removal of duplicates, 414 targets were identified as potential targets of EZP 


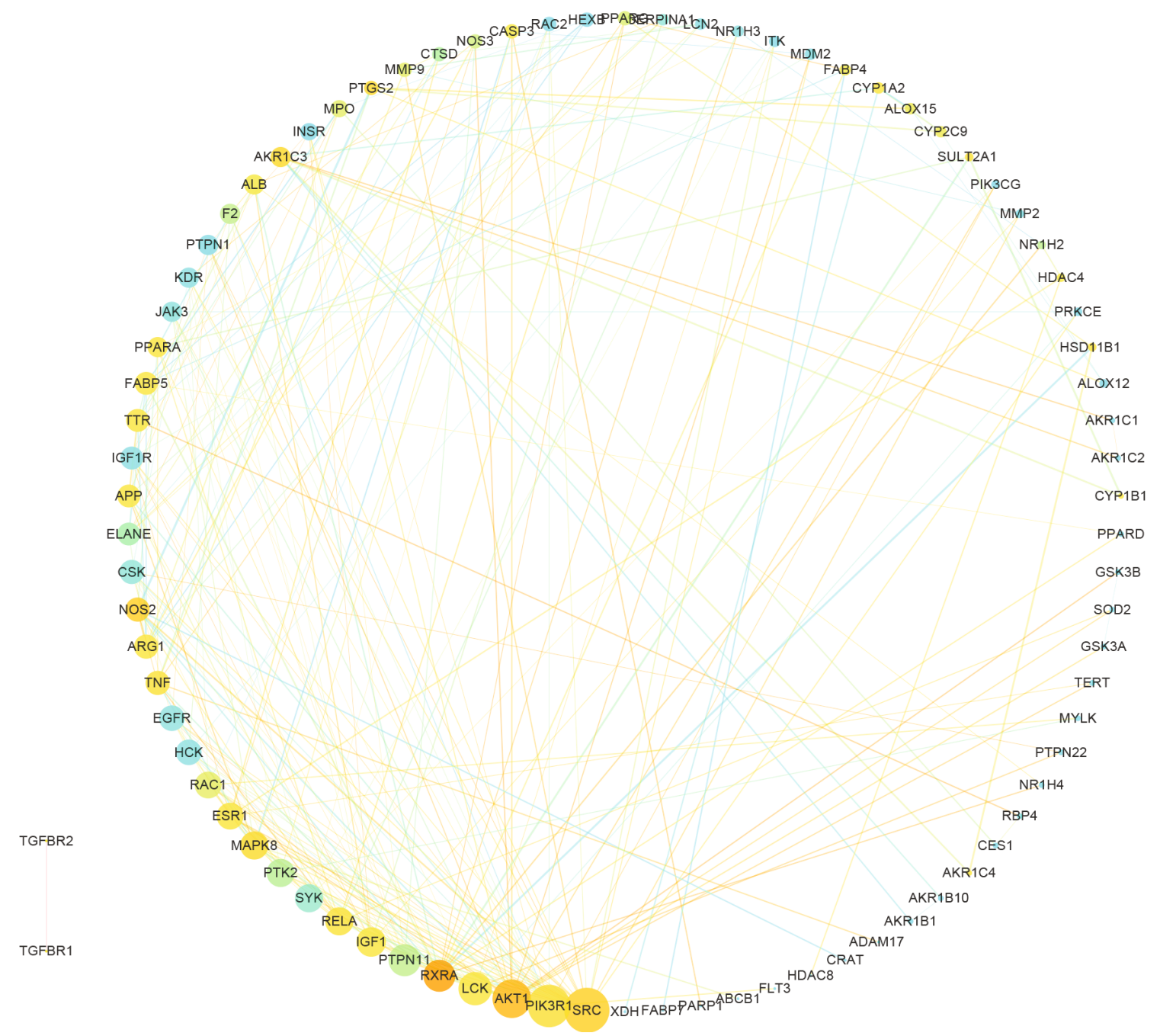

FIGURE 3: Visualization of the protein-protein interaction (PPI) using STRING and Cytoscape.

for subsequent analysis. Detailed information on EZP-related targets is shown in Table S1.

3.3. Identification of Targets Related to MAFLD. DrugBank, NCBI Gene, GeneCards, and OMIM were used to identify targets related to MAFLD. There were 313 targets from DrugBank, 161 targets from the NCBI Gene, 219 targets from GeneCards, and 149 targets from OMIM. After removal of duplicate targets, 691 targets were identified as potential therapeutic targets of MAFLD (Figure 2(b)). When overlapped with 414 targets of the EZP-related targets, 107 targets were found at the intersection of EZP-related targets and MAFLD-related targets (Figure 2(a)). Detailed information on MAFLD-related targets is presented in Table S2.

\subsection{Protein-Protein Interaction Network. STRING and} Cytoscape were used to analyze the interaction between the 107 common targets. The common targets were uploaded to STRING with limitation to "Homo sapiens" and a confidence score $>0.9$. Then, the PPI network was established and visualized by Cytoscape 3.7.1 (Figure 3), which has 82 nodes and 247 edges. Network analyzer was used to calculate topological parameters of the PPI network for identifying the hub nodes and essential targets. In Figure 3, the size and color of the node were used to describe the topological parameters of the targets. The nodes with a larger degree were described by a larger size, and the nodes with bigger between centrality were described by a darker color. The overlap of the top 20 targets of degree, between centrality and closeness centrality, LCK, MAPK8, AKT1, RXRA, PIK3R1, SRC, RELA, ESR1, NOS2, and TNF were identified as hub nodes and essential targets of the PPI network.

3.5. GO and KEGG Pathway Enrichment Analyses. DAVID was used for analyzing GO and KEGG pathway enrichment analyses to explore possible mechanisms of EZP against MAFLD by submitting 107 common targets to the database. The GO terms and KEGG pathways with $P \leq 0.05$ were significantly enriched. Biological process (BP), cell compound (CC), and molecular function (MF) are the three components of GO term enrichment analysis. The top 20 enriched results were graphed using ImageGP (Figure 4). The results 


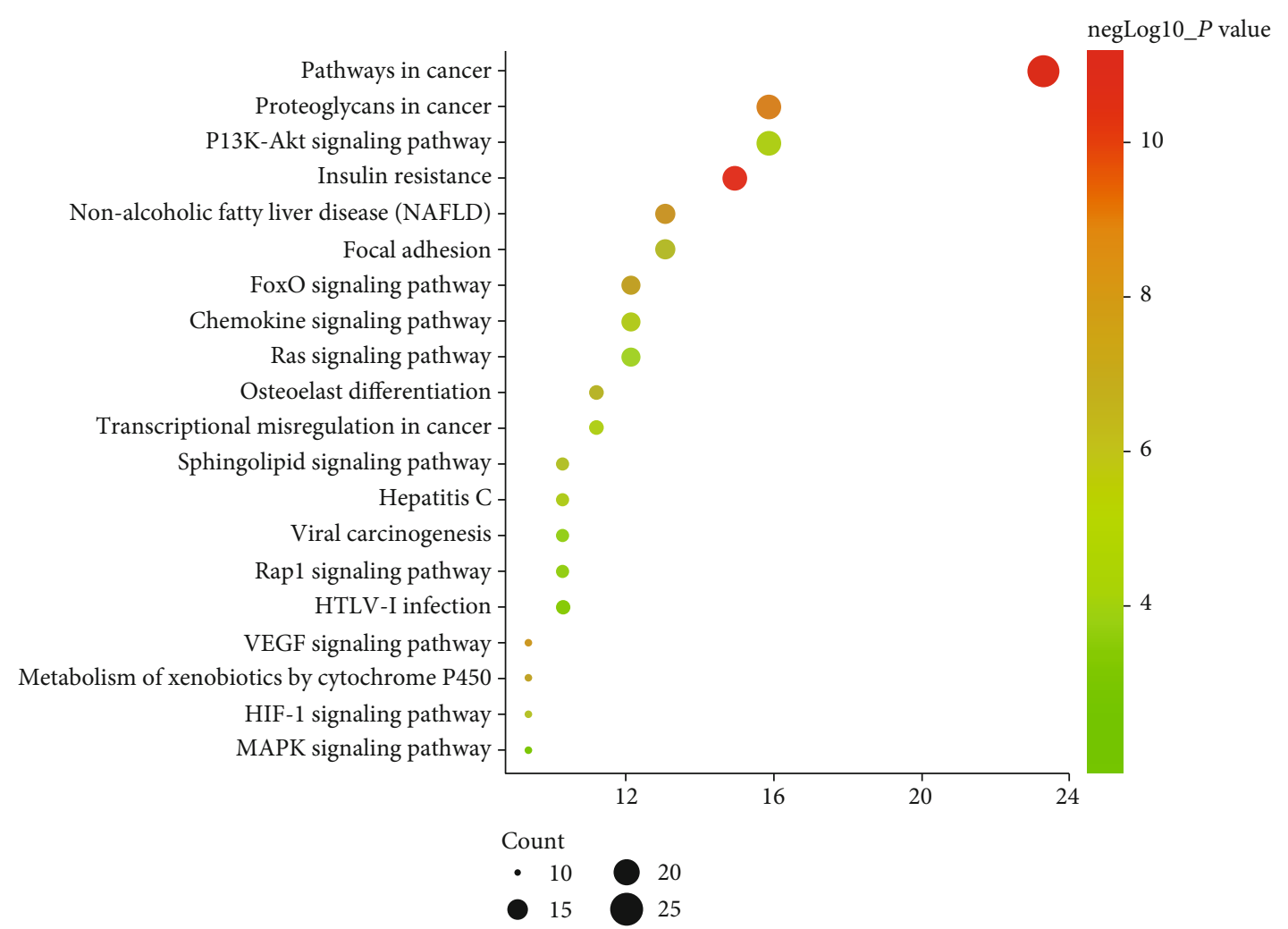

(a)

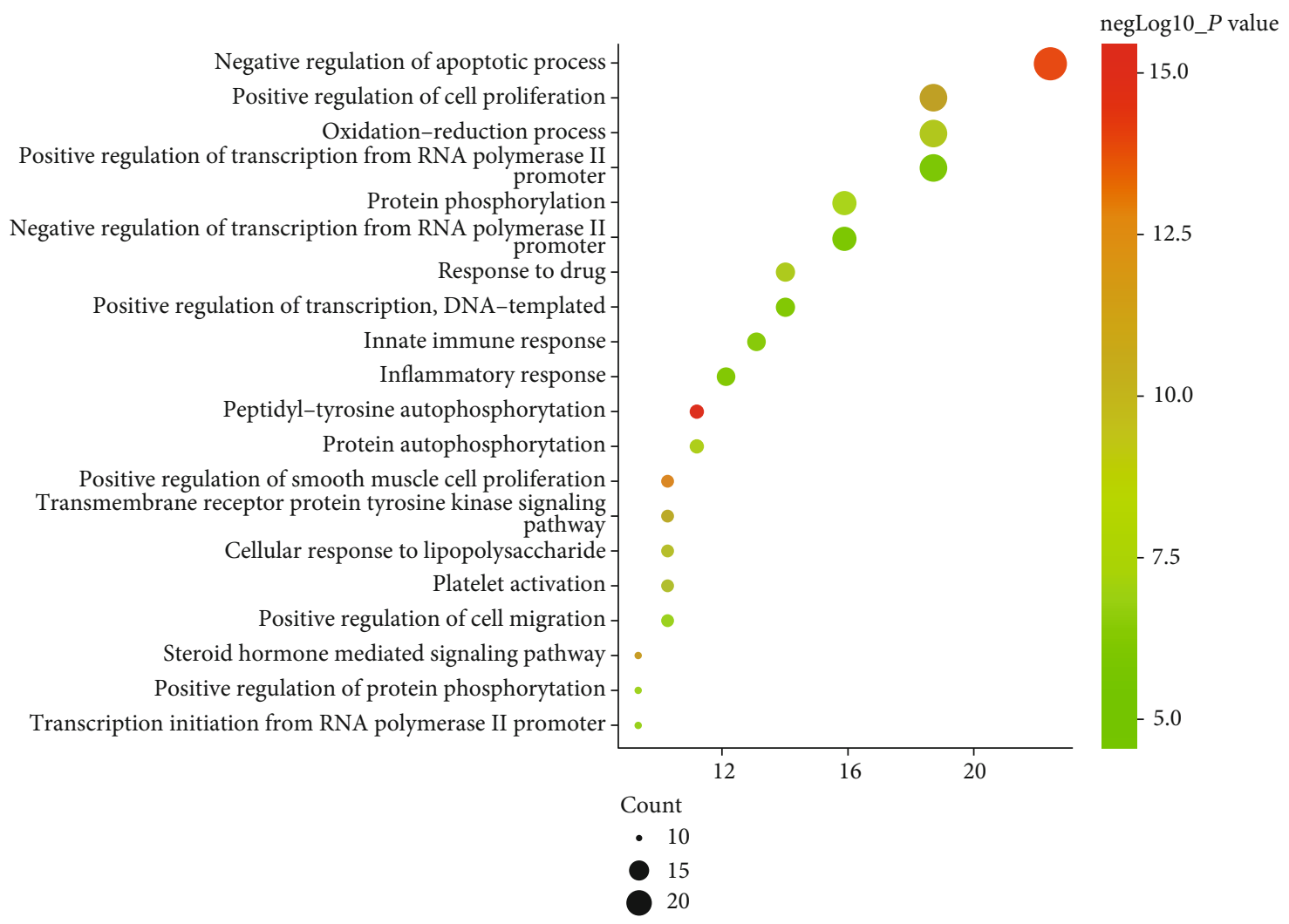

(b)

FIgURE 4: Continued. 


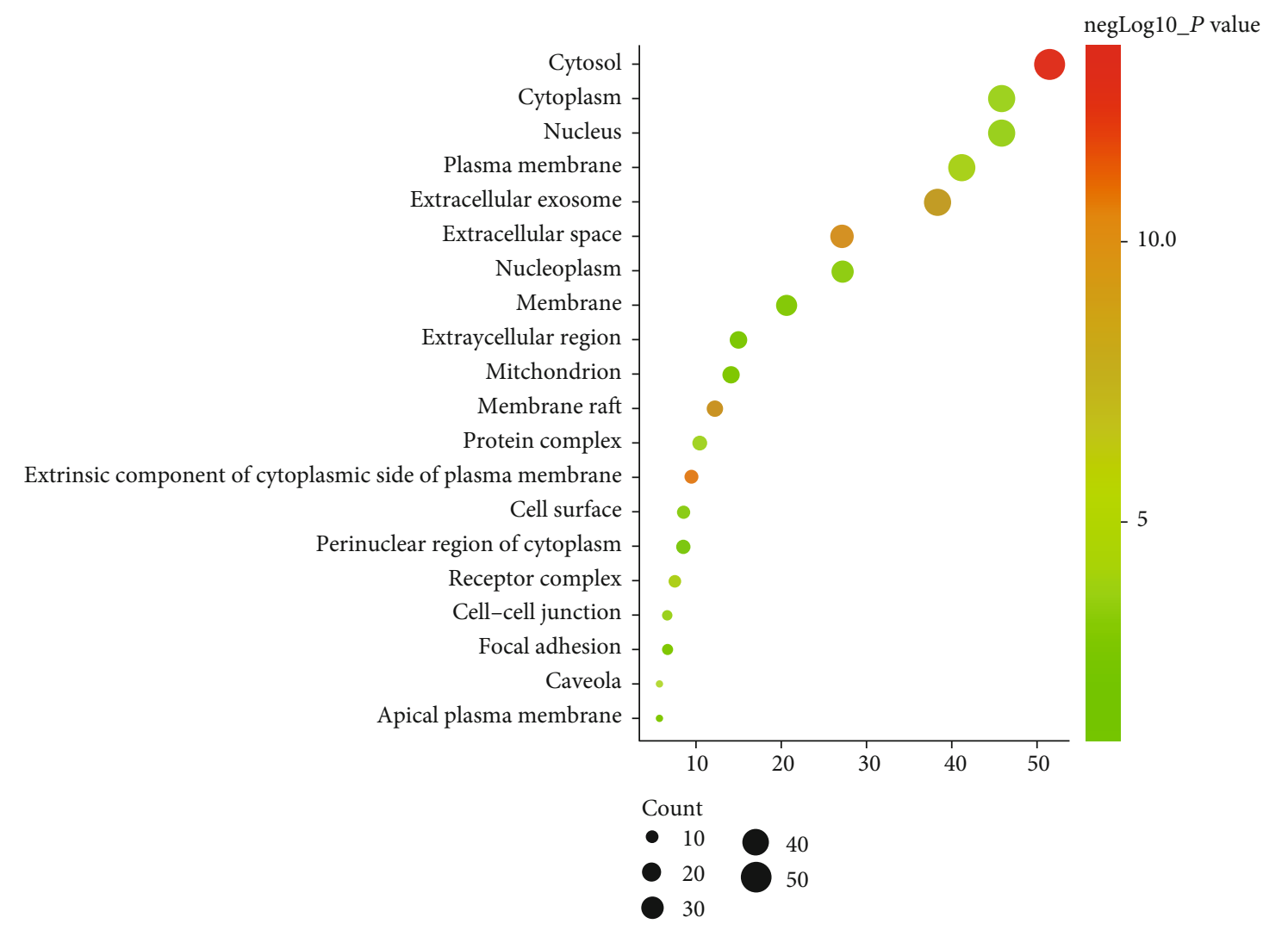

(c)

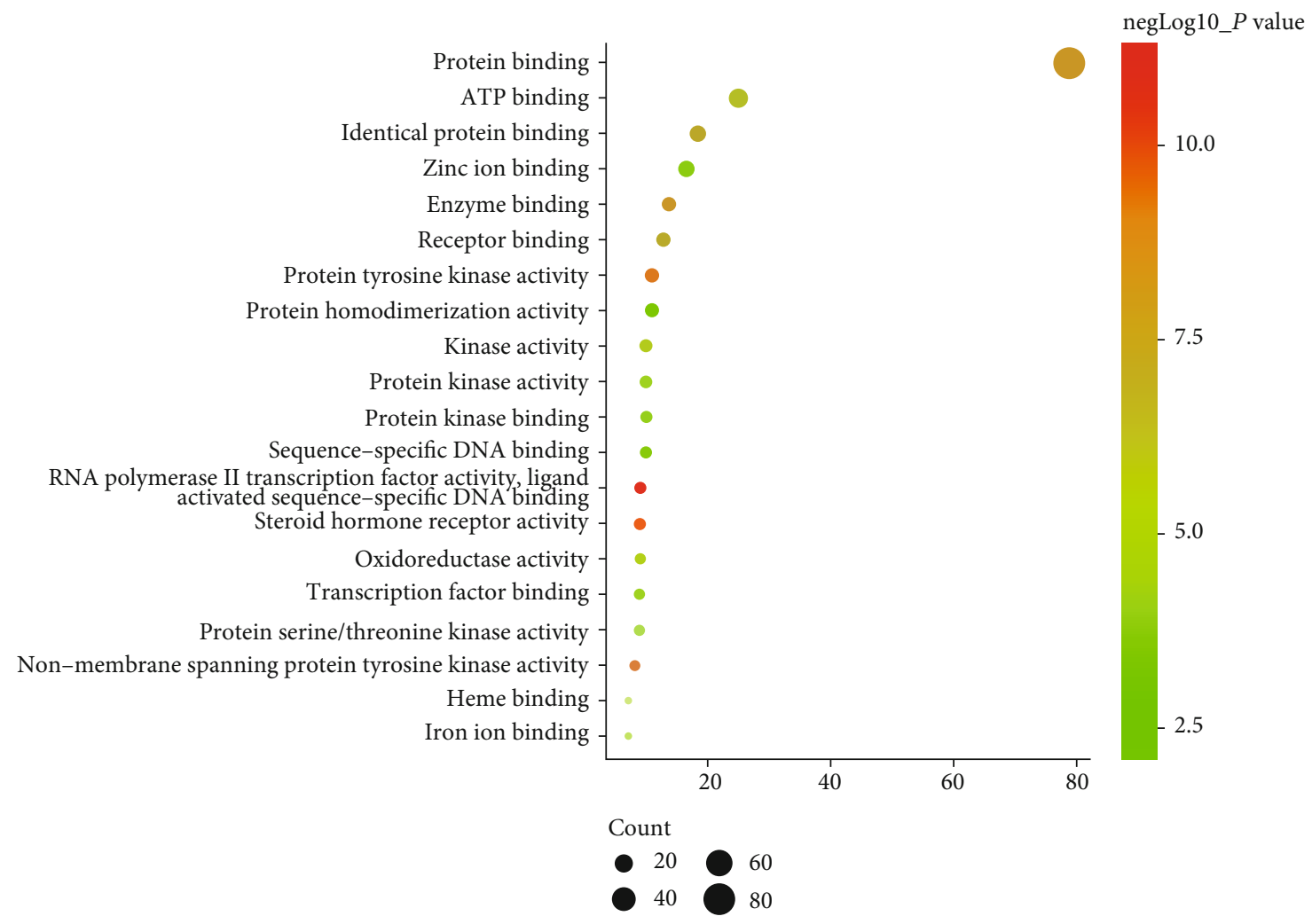

(d)

Figure 4: KEGG pathways and GO analyses. (a) KEGG pathway enrichment; (b) biological press (BP); (c) cellular component (c); (d) molecular function (MF). 


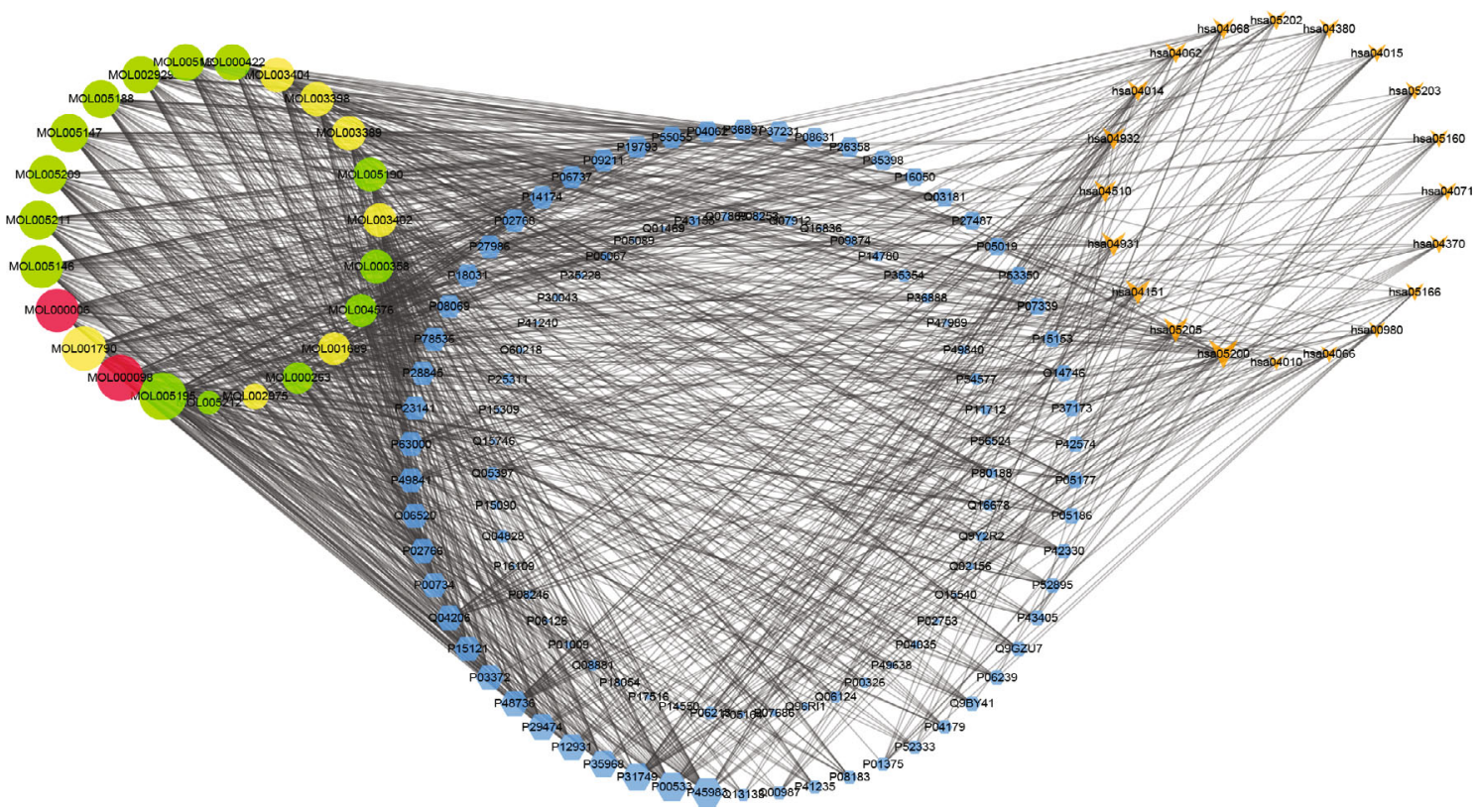

Figure 5: Compound-target-pathway network. Green circles represent bioactive components from LLF, yellow circles represent bioactive components from $\mathrm{EH}$, red circles represent duplicated components of $\mathrm{EH}$ and LLF, blue hexagons represent putative targets, and orange $\mathrm{V}$ shapes represent the top 20 pathways.

showed that 107 targets were significantly enriched in 72 BPs, 38 CCs, $103 \mathrm{MFs}$, and 83 pathways. Detailed information on GO and KEGG pathway enrichment analyses is presented in Table S3.

3.6. Construction of Compound-Target-Pathway Network. According to the GO and KEGG pathway enrichment results, a compound-target-pathway network was established by Cytoscape (Figure 5). The compound-target-pathway network included 150 nodes and 1141 edges, circles represent bioactive components from EZP, green circles represent bioactive components from LLF, yellow circles represent bioactive components from $\mathrm{EH}$, red circles represent duplicated components of $\mathrm{EH}$ and LLF, blue hexagons represent putative targets, and orange $\mathrm{V}$ shapes represent the top 20 pathway. In the compound-target-pathway network, 11 compounds had a higher than average degree, which showed that they played a pivotal role in the network. The 11 core compounds were MOL005195, MOL000098, MOL001790, MOL000006, MOL005146, MOL005211, MOL005209, MOL005147, MOL005188, and MOL002929. Targets are bridges between compounds and pathways. The interaction of the top 20 targets of the PPI network and the compound-target-pathway network was identified as core targets, which means that they play an essential role in both PPI network and compound-target-pathway network. Finally, nine targets, MAPK8, EGFR, AKT1, SRC, ESR1, RELA, RAC1, IGF1R, and PIK3R1 were identified as core targets.

3.7. Molecular Docking. Docking studies were carried out between 11 core compounds and 9 core targets to test the reliability of the drug-target interaction. These targets were chosen as core targets because they play an essential role in the top 20 KEGG pathway, but they were also core targets of the PPI network, which means that these targets may be the center of the regulatory network of EZP against MAFLD. The binding energy and grid box are shown in Table 2. The results showed that there was a stronger interaction between MOL000006, MOL000098, MOL001790, MOL005160, MOL005188, and MOL005209 and core targets. The binding energy of some docking pattern was even lower than that of the original ligand, such as MOL000006 binding with MAPK8 and MOL001790 binding with EGFR and MOL005209 binding with RELA. Figure 6 shows the docking patterns of bioactive compounds interacting with core targets in the lowest binding energy illustrated by PyMol, and the hydrogen bond is showed by a yellow imaginary line. The results showed that MOL000006 and MOL001790 have the lowest binding energy with 3 of the 9 core targets; MOL005169, MOL005188, and MOL005209 have the lowest binding energy with 1 of the 9 core targets, which means that these five compounds may have more important functions in the regulatory network of EZP against MAFLD.

\section{Discussion}

In this study, the mechanism and bioactive compounds were investigated using a bioinformatics method to investigate the hepatoprotective effects of EZP. The results showed that 83 pathways and 72 biological processes were involved. According to the topological parameters of the compoundtarget-pathway network and the PPI network, 11 bioactive 


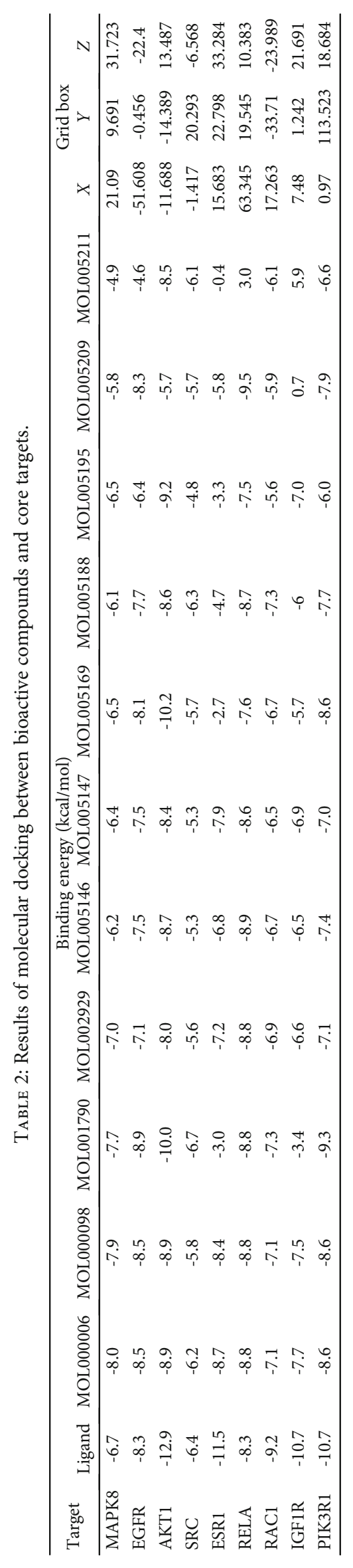




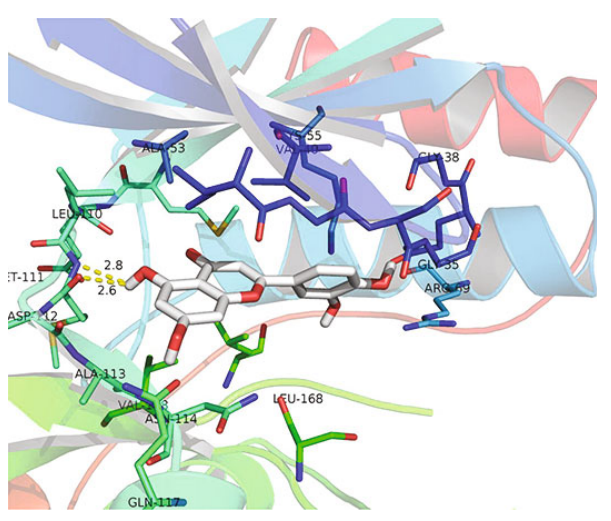

(a)

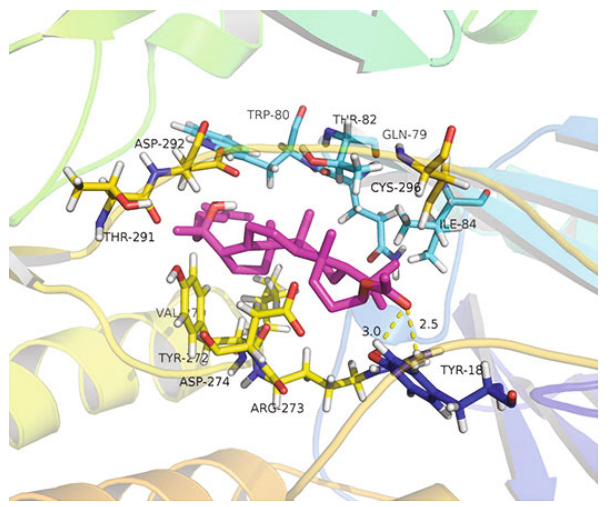

(c)

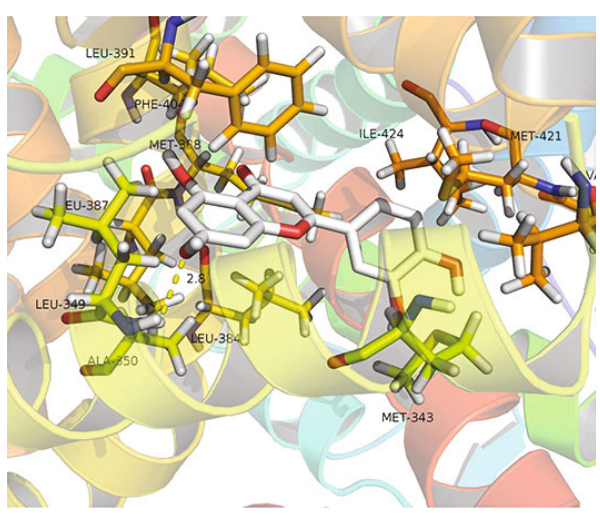

(e)

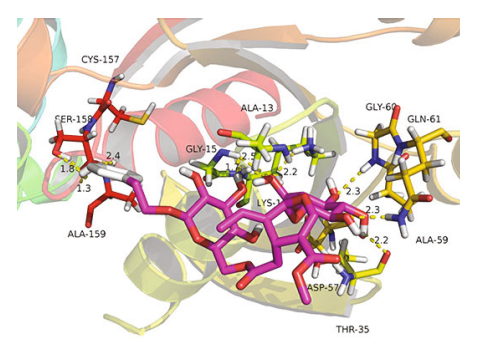

(g)

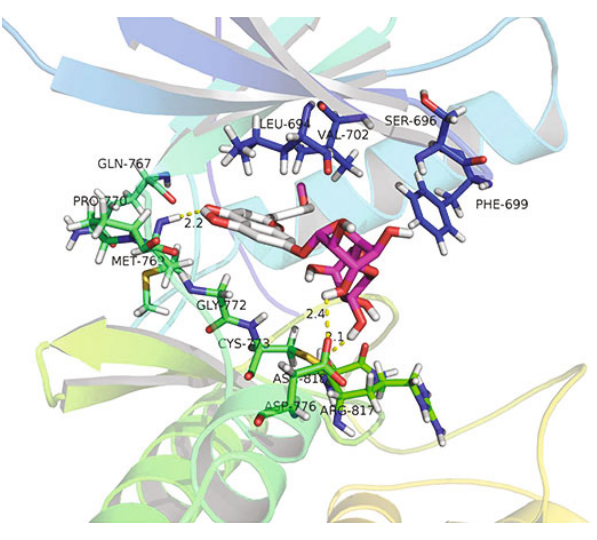

(b)

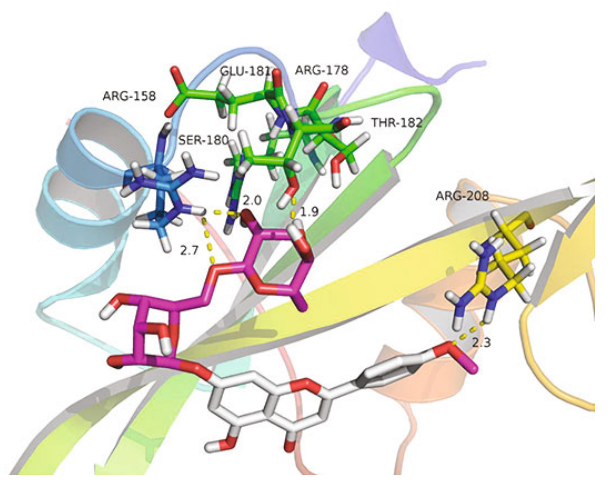

(d)

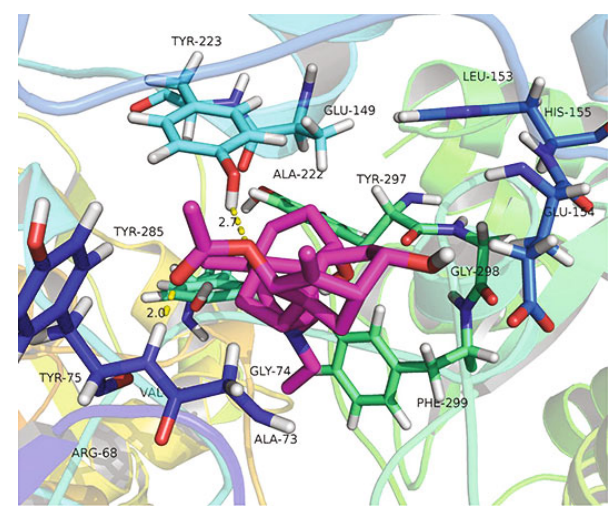

(f)

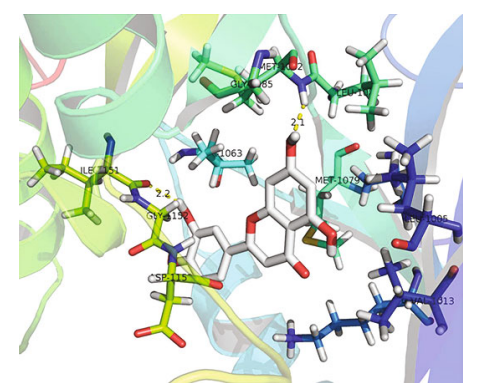

(h)

Figure 6: Continued. 


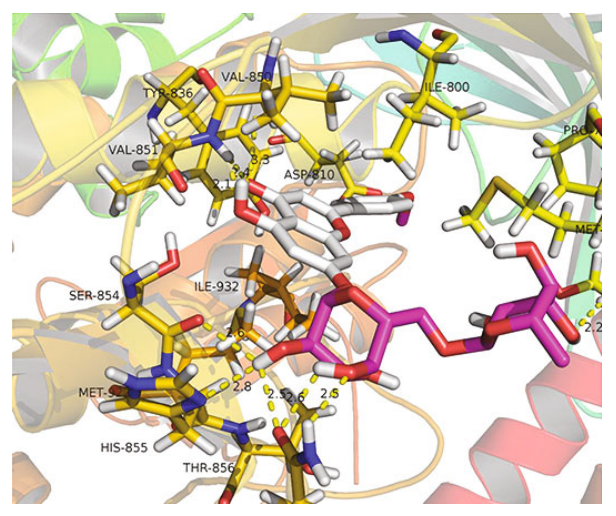

(i)

FIGURE 6: Molecular models of binding of bioactive compounds to the core targets. (a) MOL000006 to MAPK8; (b) MOL001790 to EGFR; (c) MOL005169 to AKT1; (d) MOL001790 to SRC; (e) MOL000006 to ESR1; (f) MOL005209 to RELA; (g) MOL005188 to RAC1; (h) MOL000006 to IGF1R; (i) MOL001790 to PIK3R1.

compounds and 9 core targets were identified. Finally, molecular docking was used to test the reliability of the drug-target interaction. The experimental flow is shown in Figure 7. This study could provide a better understanding of the hepatoprotective effect of EZP against MAFLD in a multicomponent and multitarget manner, which provides further insights for exploring the hepatoprotective mechanism of EZP.

In clinical treatment, EZP is administered orally. Hence, ADME-related paraments OB and DL were used for screening potential bioactive compounds of EZP. Then, the degree of potentially bioactive compounds of the compound-targetpathway network higher than average was used for a second screening. Eleven bioactive compounds were identified from EZP. To ensure the reliability of the target prediction, three different target identification databases and three multiple information sources were used to predict related targets. The PPI network and compound-target-pathway network were used to identify core targets of the regulatory network of EZP against MAFLD. The interaction of the top 20 targets of the PPI network and compound-target-pathway network was identified as a core target. Nodes with a high degree often play an essential role in the network. Core targets' degrees were higher in the PPI network and the compound-target-pathway network. This means that core targets were essential in the regulatory network of EZP against MAFLD.

The pathological mechanisms of MAFLD are complicated [35]. At present, it is a widely accepted theory that the capacity of the liver to handle the primary metabolic energy is overwhelming leading to accumulation of toxic lipid species that induce hepatocellular stress, injury, and death [35-37]. When the liver cannot handle excessive fatty acids, the excess may serve as substrates, leading to generation of lipotoxic species which would provoke ER stress and hepatocellular injury [38]. Hence, regulating fatty acid metabolism and declining hepatocellular stress, injury, and death induced by toxic lipid species are two aspects of MAFLD therapeutic strategies.
Nine core targets, MAPK8, EGFR, AKT1, SRC, ESR1 RELA, RAC1, IGF1R, and PIK3R1, were identified for molecular docking with 11 bioactive compounds. The results showed that the bioactive compounds of EZP have good affinity for nine core targets. These core targets play essential roles in the pathophysiology of MAFLD. The hsa04151: PI3K-Akt signaling pathway, in which AKT1 plays a pivotal role, was a significant result of KEGG pathway enrichment. This pathway has been proved to be closely related to the hepatoprotective effect of EZP via inhibition of hepatocyte apoptosis [39]. MAPK8 also acts a pivotal part of the development of MAFLD. During inflammation postreceptor insulin signaling is significantly impaired by MAPK8, which leads to the production of toxic lipid species and hepatocyte injury [40].

Metabolic syndrome (MetS) is the strongest risk factor for MAFLD. Among the MetS, diabetes is the clearest biological factor associated with MAFLD and 75\% of patients with type 2 diabetes have MAFLD [41]. Figure 4 shows the 16 targets involved in hsa04931: Insulin resistance. Insulin resistance is a common feature of MAFLD and leads to improper release of fatty acids further impairing insulin signaling throughout the body [42]. Molecular docking also showed that the binding energy of bioactive compounds of EZP (except lucidusculine and olitoriside with IGF1R) was lower than $-5 \mathrm{kcal} / \mathrm{mol}$, suggesting that the bioactive compounds of EZP may exert anti-MAFLD effects by insulin resistance. Figure 4 is a representation of 14 targets involved in hsa04932: Nonalcoholic fatty liver disease, which shows a stage-dependent progression of NAFLD. As shown in Figure 8, all 14 targets, marked with stars, play important roles in the progress of MAFLD, both in excess lipid accumulation and production of reactive oxygen species (ROS). This further leads to cytokine production, cell death promotion, inflammation and fibrosis. There were 14 targets enriched in hsa04932 including TNF, CASP3, MAPK, PPARA, RELA, and AKT1. These targets all play important roles in promoting cell death, inflammation, and fibrosis [43, 44], meaning that EZP may exert anti-MAFLD by these targets. 


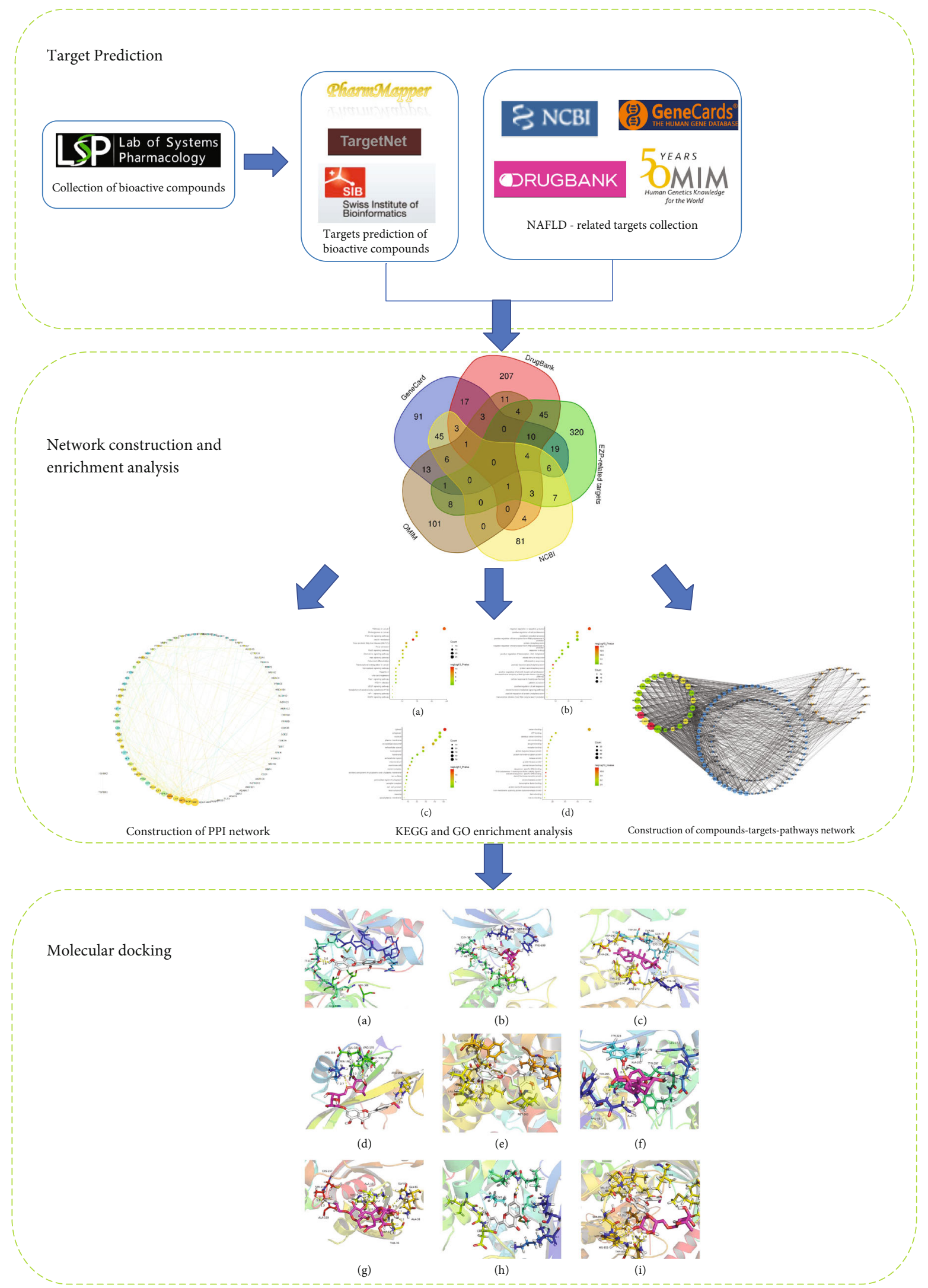

Figure 7: The experimental flow of this study. 


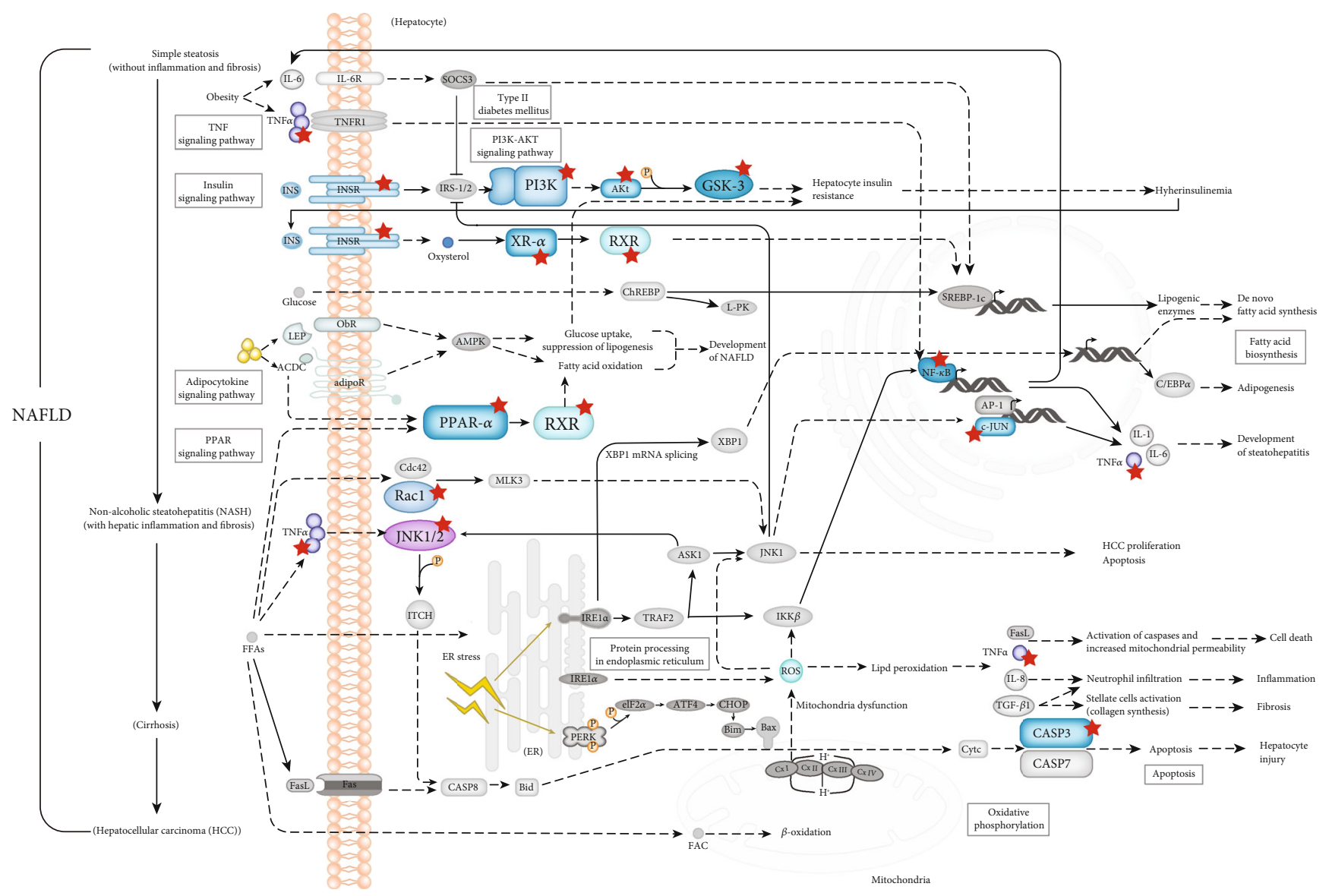

FIGURE 8: Stage-dependent progression of MAFLD. EZP-related targets are marked with red stars.

\section{Conclusion}

Overall, this study provides a theoretical basis for EZP exertion of an anti-MAFLD effect through a multicomponent, multitarget, and multipathway manner. In addition, we screened the bioactive compounds of EZP and tested them by molecular docking, providing a further understanding to explore the hepatoprotective mechanisms of EZP.

\section{Abbreviations}

EZP:

MAFLD: Metabolic dysfunction-associated fatty liver disease

NAFLD: $\quad$ Nonalcoholic fatty liver disease

PPI: $\quad$ Protein-protein interaction

NAFL: $\quad$ Nonalcoholic fatty liver

TCM: $\quad$ Traditional Chinese medicine

LLF: $\quad$ Ligustri Lucidi Fructus

EH: $\quad$ Ecliptae Herba

TCMSP: Traditional Chinese Medicine Systems Pharmacology Database

OMIM: Online Mendelian Inheritance in Man

NCBI Gene: National Centre for Biotechnology Information Gene

STRING: $\quad$ Search Tool for the Retrieval of Interacting Genes
DAVID: Database for Annotation Visualization and Integrated Discovery

GO: Gene ontology

KEGG: $\quad$ Kyoto Encyclopedia of Genes and Genomes

MAPK8: Mitogen-activated protein kinase 1

EGFR: $\quad$ Epidermal growth factor receptor

AKT1: RAC-alpha serine/threonine-protein kinase

SRC: $\quad$ Protooncogene tyrosine-protein kinase Src

ESR1: $\quad$ Estrogen receptor

RELA: Transcription factor p65

RAC1: $\quad$ Ras-related C3 botulinum toxin substrate 1

IGF1R: Insulin-like growth factor 1 receptor

PIK3R1: Phosphatidylinositol 3-kinase regulatory subunit alpha

TNF: $\quad$ Tumor necrosis factor

CASP3: $\quad$ Caspase-3

PPARA: Peroxisome proliferator-activated receptor alpha

LCK: $\quad$ Tyrosine-protein kinase Lck

RXRA: $\quad$ Retinoic acid receptor RXR-alpha

NOS2: $\quad$ Nitric oxide synthase

BP: Biological process

CC: $\quad$ Cell compound

MF: $\quad$ Molecular function

MetS: $\quad$ Metabolic syndrome

ROS: $\quad$ Reactive oxygen species. 


\section{Data Availability}

The data used to support the findings of this study are available from the corresponding author upon request.

\section{Disclosure}

The author reports no conflicts of interest in this work.

\section{Conflicts of Interest}

The authors declare that they have no conflicts of interest.

\section{Authors' Contributions}

Shaojie Huang, Fei Mu, Fei Li, and Wenjun Wang contributed equally to this work.

\section{Acknowledgments}

This study was supported by a grant from the National Natural Science Foundation of China (No. 81774190).

\section{Supplementary Materials}

Table S1: putative targets for bioactive compounds contained in EZP. There were 306 targets from PharmMapper, 156 targets from TargetNet, and 102 targets from Swiss Target Prediction. Table S2: targets related to NAFLD. Detailed information of targets related to NAFLD; there were 313 targets were from DrugBank, 161 targets from NCBI Gene, 219 targets from GeneCard, and 149 targets from OMIM. Table S3: the results of GO and KEGG pathway enrichment analyses. 107 targets were significantly enriched in $72 \mathrm{BPs}$, 38 CCs, 103 MFs, and 83 pathways. (Supplementary Materials)

\section{References}

[1] M. Eslam, A. J. Sanyal, J. George et al., "MAFLD: a consensusdriven proposed nomenclature for metabolic associated fatty liver disease," Gastroenterology, vol. 158, no. 7, pp. 19992014.e1, 2020.

[2] M. Eslam, P. N. Newsome, S. K. Sarin et al., "A new definition for metabolic dysfunction-associated fatty liver disease: an international expert consensus statement," Journal of Hepatology, vol. 73, no. 1, pp. 202-209, 2020.

[3] N. Chalasani, Z. Younossi, J. E. Lavine et al., "The diagnosis and management of nonalcoholic fatty liver disease: practice guidance from the American Association for the Study of Liver Diseases," Hepatology, vol. 67, no. 1, pp. 328-357, 2018.

[4] Z. Younossi, Q. M. Anstee, M. Marietti et al., "Global burden of NAFLD and NASH: trends, predictions, risk factors and prevention," Nature Reviews Gastroenterology \& Hepatology, vol. 15, no. 1, pp. 11-20, 2018.

[5] M. J. Armstrong, L. A. Adams, A. Canbay, and W. K. Syn, "Extrahepatic complications of nonalcoholic fatty liver disease," Hepatology, vol. 59, no. 3, pp. 1174-1197, 2014.

[6] C. D. Byrne and G. Targher, "NAFLD: a multisystem disease," Journal of Hepatology, vol. 62, no. 1, pp. S47-S64, 2015.
[7] E. Vilar-Gomez, Y. Martinez-Perez, L. Calzadilla-Bertot et al., "Weight loss through lifestyle modification significantly reduces features of nonalcoholic steatohepatitis," Gastroenterology, vol. 149, no. 2, pp. 367-378.e5, 2015.

[8] J. M. Yang, Y. Sun, M. Wang et al., "Regulatory effect of a Chinese herbal medicine formula on non-alcoholic fatty liver disease," World Journal of Gastroenterology, vol. 25, no. 34, pp. 5105-5119, 2019.

[9] B. G. Zhou, H. M. Zhao, X. Y. Lu et al., "Erzhi Pill ${ }^{\circledR}$ repairs experimental liver injury via TSC/mTOR signaling pathway inhibiting excessive apoptosis," Evidence-based Complementary and Alternative Medicine, vol. 2017, Article ID 5653643, 15 pages, 2017.

[10] A. L. Hopkins, "Network pharmacology: the next paradigm in drug discovery," Nature Chemical Biology, vol. 4, no. 11, pp. 682-690, 2008.

[11] R. Zhang, X. Zhu, H. Bai, and K. Ning, "Network pharmacology databases for traditional Chinese medicine: review and assessment," Frontiers in Pharmacology, vol. 10, p. 123, 2019.

[12] W. Wu, Z. Zhang, F. Li et al., "A network-based approach to explore the mechanisms of Uncaria alkaloids in treating hypertension and alleviating Alzheimer's disease," International Journal of Molecular Sciences, vol. 21, no. 5, article 1766, 2020.

[13] J. Zhang, H. Li, Y. Zhang, C. Zhao, Y. Zhu, and M. Han, "Uncovering the pharmacological mechanism of stemazole in the treatment of neurodegenerative diseases based on a network pharmacology approach," International Journal of Molecular Sciences, vol. 21, no. 2, p. 427, 2020.

[14] J. Ru, P. Li, J. Wang et al., "TCMSP: a database of systems pharmacology for drug discovery from herbal medicines," Journal of Cheminformatics, vol. 6, no. 1, p. 13, 2014.

[15] X. Xu, W. Zhang, C. Huang et al., "A novel chemometric method for the prediction of human oral bioavailability," International Journal of Molecular Sciences, vol. 13, no. 6, pp. 6964-6982, 2012.

[16] W. P. Walters and M. A. Murcko, "Prediction of 'drug-likeness'," Advanced Drug Delivery Reviews, vol. 54, no. 3, pp. 255-271, 2002.

[17] Z. J. Yao, J. Dong, Y. J. Che et al., "TargetNet: a web service for predicting potential drug-target interaction profiling via multi-target SAR models," Journal of Computer-Aided Molecular Design, vol. 30, no. 5, pp. 413-424, 2016.

[18] X. Wang, Y. Shen, S. Wang et al., "PharmMapper 2017 update: a web server for potential drug target identification with a comprehensive target pharmacophore database," Nucleic Acids Research, vol. 45, no. W1, pp. W356-W360, 2017.

[19] X. Wang, C. Pan, J. Gong, X. Liu, and H. Li, "Enhancing the enrichment of pharmacophore-based target prediction for the polypharmacological profiles of Drugs," Journal of Chemical Information and Modeling, vol. 56, no. 6, pp. 1175-1183, 2016.

[20] A. Daina, O. Michielin, and V. Zoete, "SwissTargetPrediction: updated data and new features for efficient prediction of protein targets of small molecules," Nucleic Acids Research, vol. 47, no. W1, pp. W357-W364, 2019.

[21] D. Gfeller, O. Michielin, and V. Zoete, "Shaping the interaction landscape of bioactive molecules," Bioinformatics, vol. 29, no. 23, pp. 3073-3079, 2013.

[22] X. Liu, S. Ouyang, B. Yu et al., "PharmMapper server: a web server for potential drug target identification using 
pharmacophore mapping approach," Nucleic Acids Research, vol. 38, pp. W609-W614, 2010.

[23] D. S. Wishart, Y. D. Feunang, A. C. Guo et al., "DrugBank 5.0: a major update to the DrugBank database for 2018," Nucleic Acids Research, vol. 46, no. D1, pp. D1074-D1082, 2018.

[24] J. S. Amberger and A. Hamosh, "Searching Online Mendelian Inheritance in Man (OMIM): a knowledgebase of human genes and genetic phenotypes," Current Protocols in Bioinformatics, vol. 58, pp. 1-2, 2017.

[25] S. Fishilevich, S. Zimmerman, A. Kohn et al., "Genic insights from integrated human proteomics in GeneCards," Database: The Journal of Biological Databases and Curation, vol. 2016, 2016.

[26] E. W. Sayers, J. Beck, J. R. Brister et al., "Database resources of the national center for biotechnology information," Nucleic Acids Research, vol. 48, no. D1, pp. D9-D16, 2020.

[27] D. Szklarczyk, J. H. Morris, H. Cook et al., "The STRING database in 2017: quality-controlled protein-protein association networks, made broadly accessible," Nucleic Acids Research, vol. 45, no. D1, pp. D362-D368, 2017.

[28] P. Shannon, A. Markiel, O. Ozier et al., "Cytoscape: a software environment for integrated models of biomolecular interaction networks," Genome Research, vol. 13, no. 11, pp. 24982504, 2003.

[29] H. Chen, N. Wang, X. Zhao, C. A. Ross, K. S. O'Shea, and M. G. McInnis, "Gene expression alterations in bipolar disorder postmortem brains," Bipolar Disorders, vol. 15, no. 2, pp. 177-187, 2013.

[30] M. Kanehisa and Y. Sato, "KEGG mapper for inferring cellular functions from protein sequences," Protein Science, vol. 29, no. 1, pp. 28-35, 2020.

[31] M. Kanehisa, "Toward understanding the origin and evolution of cellular organisms," Protein Science, vol. 28, no. 11, pp. 1947-1951, 2019.

[32] O. Trott and A. J. Olson, "AutoDock Vina: improving the speed and accuracy of docking with a new scoring function, efficient optimization, and multithreading," Journal of Computational Chemistry, vol. 31, no. 2, pp. 455-461, 2010.

[33] L. Jia, L. Fu, X. Wang et al., "Systematic profiling of the multicomponents and authentication of Erzhi Pill by UHPLC/Q-Orbitrap-MS oriented rapid polarity-switching data-dependent acquisition and selective monitoring of the chemical markers deduced from fingerprint analysis," Molecules, vol. 23, no. 12, article 3143, 2018.

[34] Y. Nie and W. Yao, "A comprehensive quality evaluation method based on C30-HPLC and an analytic hierarchy process for the Chinese herbal formula, Erzhiwan," Molecules, vol. 23, no. 8, article 2045, 2018.

[35] C. Alonso, D. Fernández-Ramos, M. Varela-Rey et al., "Metabolomic identification of subtypes of nonalcoholic steatohepatitis," Gastroenterology, vol. 152, no. 6, pp. 1449-1461.e7, 2017.

[36] P. Hirsova, S. H. Ibrabim, G. J. Gores, and H. Malhi, "Lipotoxic lethal and sublethal stress signaling in hepatocytes: relevance to NASH pathogenesis," Journal of Lipid Research, vol. 57, no. 10, pp. 1758-1770, 2016.

[37] J. Boursier, O. Mueller, M. Barret et al., "The severity of nonalcoholic fatty liver disease is associated with gut dysbiosis and shift in the metabolic function of the gut microbiota," Hepatology, vol. 63, no. 3, pp. 764-775, 2016.

[38] S. L. Friedman, B. A. Neuschwander-Tetri, M. Rinella, and A. J. Sanyal, "Mechanisms of NAFLD development and thera- peutic strategies," Nature Medicine, vol. 24, no. 7, pp. 908-922, 2018.

[39] H. M. Zhao, X. Y. Zhang, X. Y. Lu et al., "Erzhi Pill ${ }^{\circledR}$ protected experimental liver injury against apoptosis via the PI3K/Akt/Raptor/Rictor pathway," Frontiers in Pharmacology, vol. 9, p. 283, 2018.

[40] M. Pal, M. A. Febbraio, and G. I. Lancaster, "The roles of c-Jun $\mathrm{NH} 2$-terminal kinases (JNKs) in obesity and insulin resistance," The Journal of Physiology, vol. 594, no. 2, pp. 267279, 2016.

[41] A. J. Käräjämäki, R. Bloigu, H. Kauma et al., "Non-alcoholic fatty liver disease with and without metabolic syndrome: different long-term outcomes," Metabolism, vol. 66, pp. 55-63, 2017.

[42] R. Loomba, M. Abraham, A. Unalp et al., "Association between diabetes, family history of diabetes, and risk of nonalcoholic steatohepatitis and fibrosis," Hepatology, vol. 56, no. 3, pp. 943-951, 2012.

[43] A. J. Sanyal, N. Chalasani, K. V. Kowdley et al., "Pioglitazone, vitamin E, or placebo for nonalcoholic steatohepatitis," The New England Journal of Medicine, vol. 362, no. 18, pp. 1675$1685,2010$.

[44] S. E. Mahady, A. C. Webster, S. Walker, A. Sanyal, and J. George, "The role of thiazolidinediones in non-alcoholic steatohepatitis - a systematic review and meta analysis," Journal of Hepatology, vol. 55, no. 6, pp. 1383-1390, 2011. 